\title{
Improving diagnostics of damage to railway catenary supports
}

\author{
Valeriy $\mathrm{Li}^{1}$, Lyudmila Demina ${ }^{1}$, and Sergey Vlasenko ${ }^{1, *}$ \\ ${ }^{1}$ Far Eastern State Transport University, 47, Seryshev Str., Khabarovsk 680021, Russia
}

\begin{abstract}
The inspection of reinforced concrete catenary supports is a time-consuming process that necessitates a lot of time to inspect the entire fleet of supports. A large number of supports with a service life of more than 40 years require intensive monitoring and condition forecasting, which can inevitably lead to high economic costs for the work of the catenary electro-mechanics. The introduction of special technical tools to collect and process measurements could solve this problem and allow the planning of a replacement schedule for the supports based on their actual damage. The article discusses the basic factors affecting the load-bearing capacity of rein-forced concrete supports, the main damages occurring, and the procedure for carrying out inspections and assessing the current condition of the supports.
\end{abstract}

\section{Introduction}

Currently, more than $63 \%$ of the total fleet of reinforced concrete catenary supports in the electrified railways exceeds the norm of 40 years, and a fifth exceeds the 50 -year limit, which multiplies the risk of damage and can therefore lead to catenary failure and train downtime [1]. The exploitation of supports with a ser-vice life of more than 40 years is an issue in seven directorates. The proportion of defective supports in certain directorates, including the Far East, has the highest number. It is not viable to increase the expense of replacing all damaged supports in the fleet of supports. As a result, the task of evaluating the support fleet in terms of defect severity becomes vital [2].

\section{Materials and methods}

Reinforced concrete structures require special attention and more in-depth scrutiny because of the peculiarities of the continuous physical process of damage and crack accumulation in concrete [3]. The slow and lengthy process of accumulation, development, and propagation of cracks on their surface is a precursor to sudden failure and collapse in operation, and therefore a threat to train traffic safety [4].

The concrete used in the construction of the catenary supports, in addition to the usual requirements for strength, is required to meet the long-term operational reliability under a wide range of climatic conditions.

*Corresponding author: vsa_ens@mail.ru 
The structure of external influences is determined by some factors that affect the support while in use. These include static loads from the weight of the overhead line catenary, overhead line wires, switching and protective equipment; vibrodynamic effects from wind and rolling stock (aerodynamic front, ground vibrations); ambient temperature and humidity; corrosive effects of soil and atmosphere; corrosion of concrete; electrical corrosion caused by leakage currents, especially on electrified DC railways [5].

All of the above factors harm supports. Static loads, dynamic effects from wind and rolling stock, and temperature effects all contribute to changes in the stress state of the material of supports [6]. Exposure to leakage currents, corrosive soil and air effects alter the physical and mechanical properties of the material of supports. Long-lasting exposure to some effects or an unfavorable combination of others can lead to defects and, consequently, a lower load-bearing capacity of supports.

The aforementioned impacts result in damage to the supports, reducing their overall mechanical strength. We suggest their classification in Fig. 1.

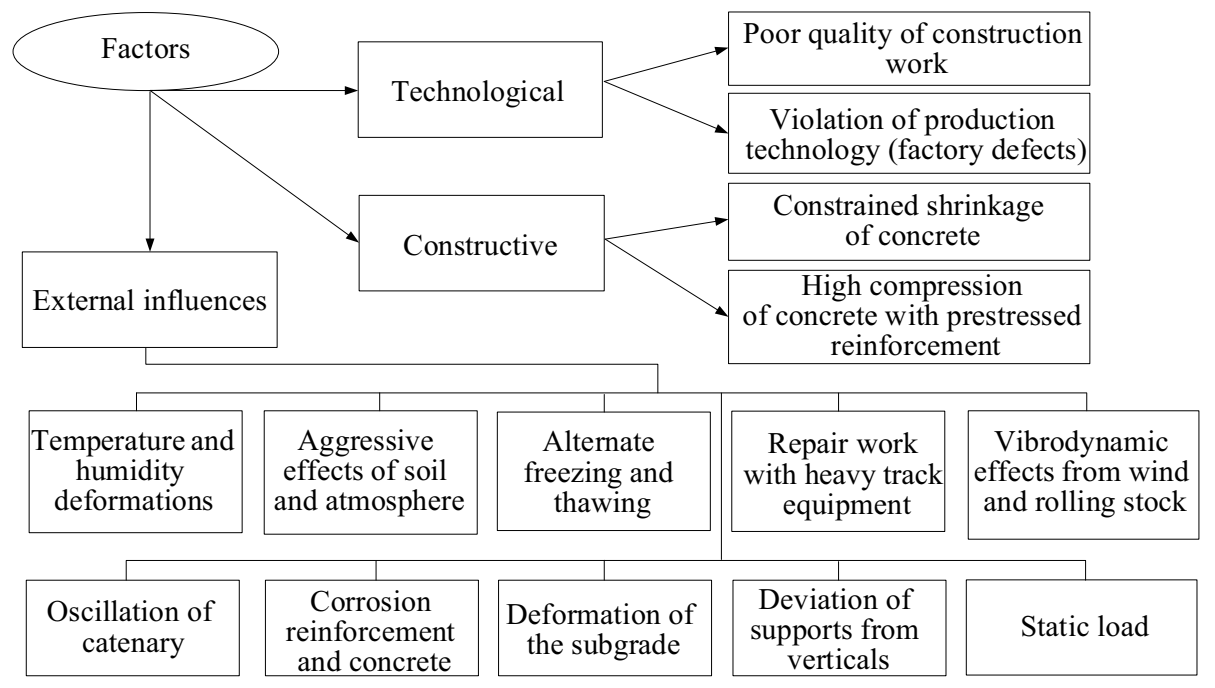

Fig. 1. Factors affecting the load-bearing capacity of reinforced concrete catenary supports.

The mechanisms of destructive processes must be considered, the probability and approximate rate of their occurrence must be estimated, and the territorial location of a particular type of failure must be determined to enable informed decisions to be made in operating conditions to predict the failure and risk of the structures in service. The analysis must take into account the most common types of failure that occur in service, such as atmospheric-soil corrosion of concrete and reinforcement, electrical corrosion failure, and the appearance and spread of cracks in the supports (Fig. 2).

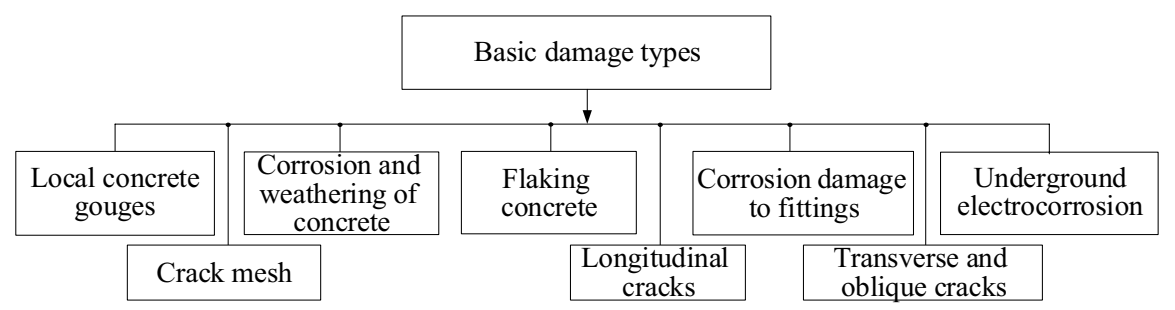

Fig. 2. Basic damage types that occur in reinforced concrete supports. 
The corrosion resistance of concrete supports is discussed in detail in [7]. The composition and structure of concrete play a major role in its corrosion rate. Sulfates and chlorides found in natural waters cause the most severe corrosive damage to concrete.

It is nearly impossible to take any broad measures to slow down the destructive processes that occur when the supports are in use. This should be done during the design, fabrication, and installation phase of the structures; during operation, the progress of corrosion processes should be monitored and the structures deteriorating should be replaced if necessary [8]. To avoid an emergency condition, the types and degree of corrosiveness of the environment should be investigated at the start of the operation, as well as the nature of any potential failure and its consequences [9].

The corrosion of concrete reinforcement is an electrochemical process. This is primarily due to the thin protective layer on the inside of annular cross-section supports, which is most often not found at the tops of the supports. This is caused by the longitudinal displacement of the concrete when it is centrifuged. As a result, the structure's strength is reduced due to a reduction in the cross-section of the reinforcement, embrittlement, and loss of adhesion with the concrete in places where the protective layer is peeled off.

The greatest danger of all external effects is electrical corrosion (on DC railways) of the reinforcement of the support footings caused by currents flowing off the rails [10]. The authors of [11] established the relationship between electrical corrosion and mechanical state of the support, determined the electrochemical parameters of concrete and reinforcement affecting the occurrence of corrosion processes, determined the natural environment effects, developed and recommended methods of corrosion measurements and corrosion protection. However, despite the precautions taken to protect support structures from rail currents, electro-corrosion damage cannot be completely avoided.

Multiple in-service observations of the condition of the catenary supports have led to the classification that the main damage issues for the catenary supports are cracks in the concrete, both longitudinal and transverse. They can cause supports to fracture and fall, causing overhead wires to break and disrupting power supply and traffic patterns for trains on the affected section of the railway.

Under the influence of climatic and force factors, the catenary supports experience significant tensile stresses, which can combine in unfavorable values and cause cracks (Fig. 3). The reasons for cracking in reinforced concrete supports are now pretty well understood [12]. However, it is worth mentioning that the crack-generating factors revealed additionally in the studies, such as the thermo-mechanical effect generated by solar insolation and the electromagnetic field of a catenary, require special consideration.

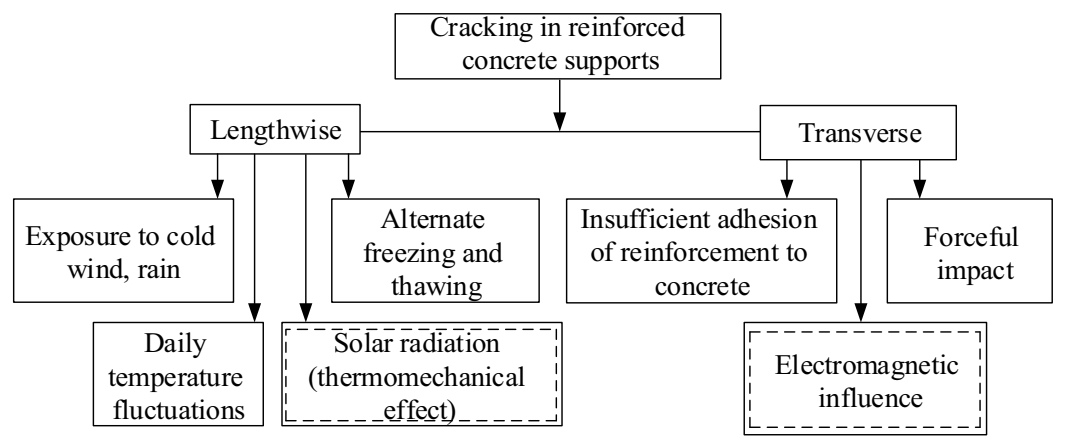

Fig. 3. Major causes of the occurrence and spread of cracks.

According to the analysis data, longitudinal cracks in supports are the most typical type of damage. The effect of natural and climatic factors that cause temperature changes in the body of the support, leading to stresses in the surface layers of the concrete, reducing the 
tensile strength of the concrete, is the main reason for the appearance of longitudinal cracks in supports during the operational period.

The complex of environmental temperature effects on the catenary supports is quite large, although it does not occur in a consistent or timely manner.

Temperature stresses are 2.5 times lower than shrinkage stresses [13]. As such, these stresses can cause the longitudinal cracks to build up in the supports, and when combined with other effects, accelerate the fracture processes, reducing the strength of the structure.

The concrete quality of the catenary supports operating in constant temperature environments has been reported to be decreasing. Specifically, when the supports are subjected to alternating freezing and thawing in the autumn and spring, structural changes in the concrete occur, the modulus of elasticity drops, and the concrete loses strength. Visual inspection in the studied area (Appendix A) revealed longitudinal cracks on the north side of the supports (over 90\%), confirming this fact. It was also established that solar insolation affects crack formation due to unequal heating on the southern and northern sides of the supports (temperature differences of up to $35^{\circ}$ ).

When a train moves (especially at high speeds), it blows on the support surface, causing a significant shift in the conditions of heat exchange between the support and the environment. Additional tensile stresses are formed as a result of temperature differences, causing the concrete to become increasingly micro-cracked.

[14] provides an example of the use of supports (lattice, I-beam) with vents to reduce temperature differences between the outside and inside surfaces. However, as previously stated, they were not widely used and were eventually replaced due to their high damageability.

Longitudinal cracks in supports can be caused by frost heaving in the interior concrete layers after many years of service. Based on the research findings, the structure and density of concrete, the degree of water saturation and the chemical composition of dissolved substances, the freezing temperature, and the rate at which it decreases are the main factors that determine the frost resistance of concrete [15]. Concrete loses its strength properties and becomes delaminated as a result of repeated freezing and thawing. Therefore, special attention needs to be paid to the condition of the concrete component, especially in the sharply varying temperature profiles of the Far East [16].

No radical measures have yet been suggested to improve the frost resistance of concrete in service. They have to be taken during the structural fabrication phase, primarily by selecting the appropriate concrete composition [17].

The most common belief is that transverse cracks are caused by force factors. These can also be caused by insufficient adhesion between the reinforcement and the concrete. Opening of such cracks more than $0.5 \mathrm{~mm}$ is not permissible [12].

Force damage happens when the design does not account for actual loads when design variations occur during manufacture, and when the weight on the structure is raised by hanging more supply and reinforcement wires, installing brackets, or other similar factors.

Support cracking occurs differently in stressed and unstressed reinforcement. For this reason, while designing centrifugal supports, the degree of prestressing of the reinforcement is critical [18]. High-strength wire reinforcement has the greatest degree of prestressing due to its high standard tensile strength. It has controlled stresses of 800 to $900 \mathrm{MPa}$, as measured at the end of tensioning on the stops. Because of the high tension level in the reinforcement, a large portion of the pre-stressing of the reinforcement is not relieved when the concrete reaches the strength limit state; rather, it stays in tension and contributes to accelerated fracture of the concrete when it reaches the strength limit state. One of the main reasons for the high sensitivity of the load-bearing capacity of wire-reinforced supports to changes in concrete strength is the presence of unrelieved prestresses in the limit state when compressive stresses reach the values equivalent to the concrete strength. 


\section{Results and discussion}

The assessment of the residual load-bearing capacity of reinforced concrete supports used in power grids of various applications is quite a complex task, as some defects are latent and the complex physical and chemical combination of reinforcement and concrete requires a comprehensive approach to this challenge [19]. For example, it is nearly impossible to adequately measure both the degree of decline in load-bearing capacity of supports owing to aging concrete and the degree of corrosion of the reinforcement in the support body without the use of destructive testing procedures. A major challenge also arises when it comes to assessing the quality of the concrete and reinforcement in the underground part of the support [20].

Based on the research completed, a methodology for predicting the condition of catenary supports has been derived [1], enabling the current condition of catenary supports to be assessed and their remaining service life to be identified.

To automate the collecting and processing of research data, an algorithm for the operation of technical tools must be developed, as well as a list of required measures to be applied in the power supply divisions.

In the long run, condition assessment and maintenance of reinforced concrete supports are critical. Visual inspection is a commonly used method where surface defects are visually observed and a knowledge- and measurement-based assessment is made and entered into a special card (datasheet) to a support. This simple method is incapable of detecting hidden damage or predicting long-term changes in the strength characteristics of supports. For more precise measurements, a variety of destructive and non-destructive methods are used.

A graphical representation of the damage to the support can be used as input information for determining the condition of the supports. The data needed for analysis should be consolidated in a special database that can be used for further analysis and decision-making.

The main anchoring element must be identified by category, e.g. cracking of the support surface (Fig. 4).
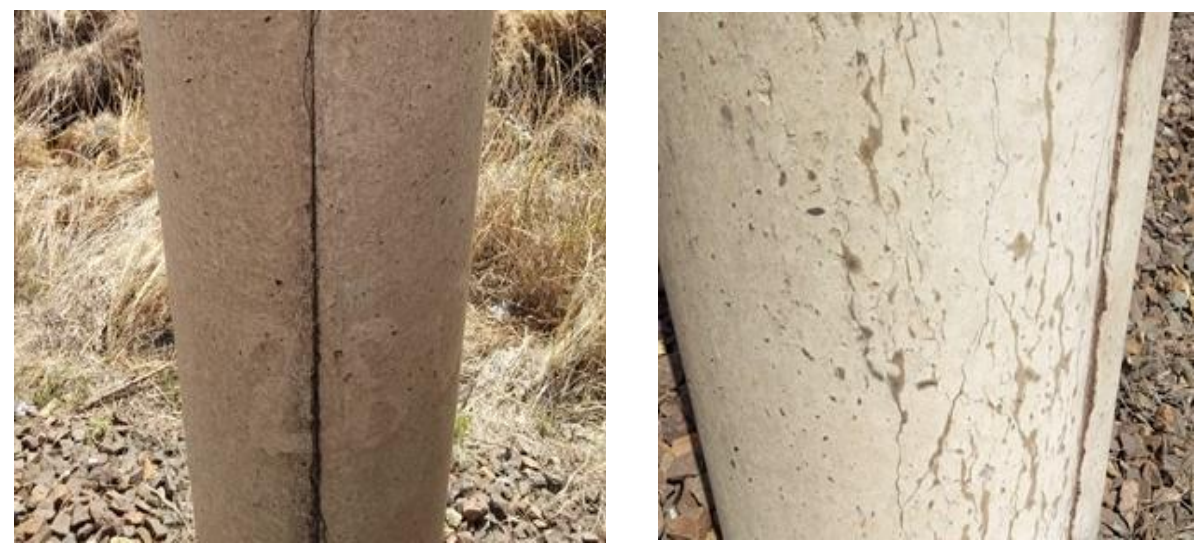

Fig. 4. Cracking of the support surface.

Images of the supports from electrical installation teams can be used to determine the current level of damage. A manual calculation of the fatigue rate and modeling of wear and damage progression, on the other hand, will significantly slow down the maintenance and repair process [21]. Automation of these operations is required, which will determine how data entry, calculation, and defect remediation measures will be carried out (Fig. 5). 


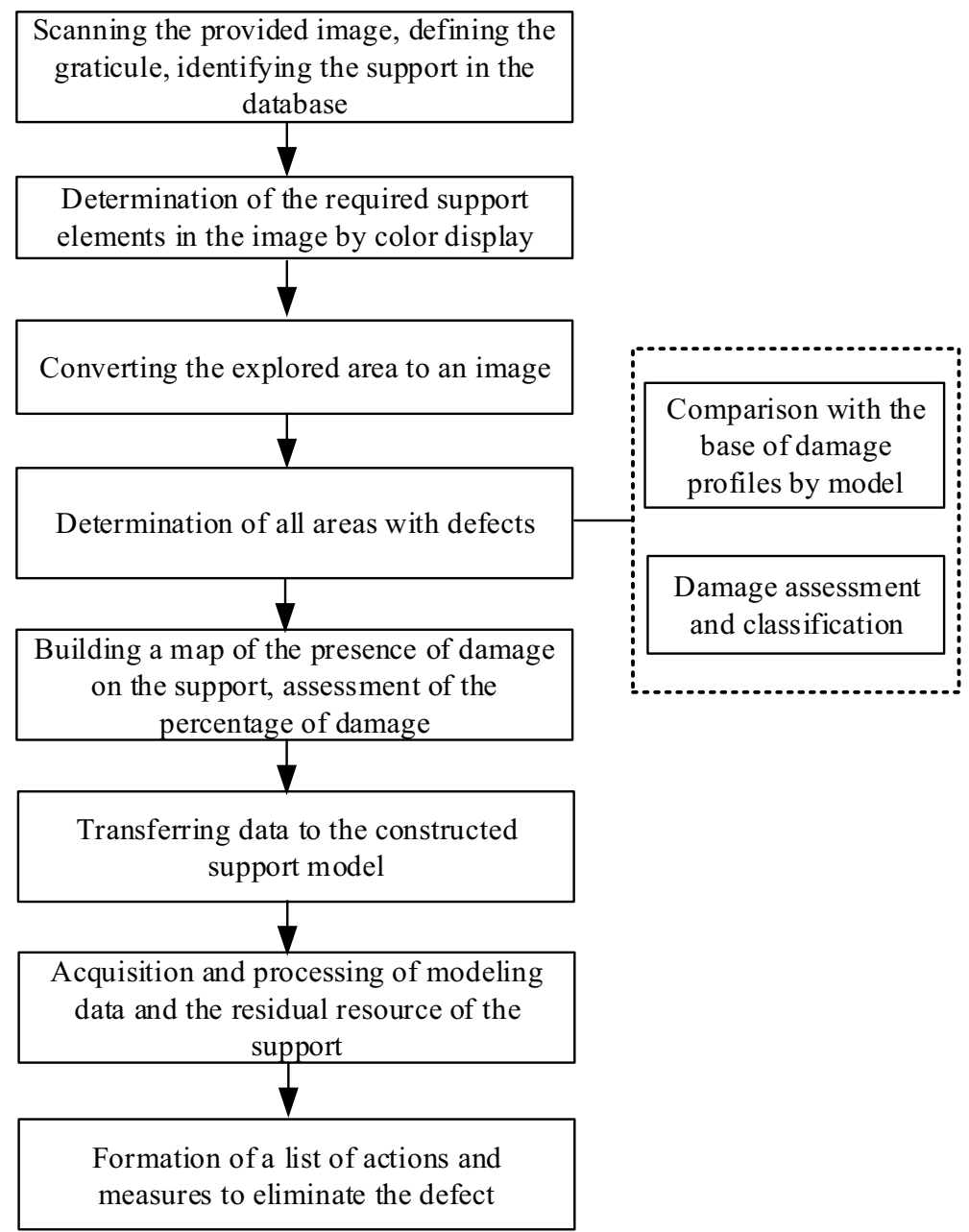

Fig. 5. Procedure for the processing of survey results.

Image processing is accompanied by filtering of the image and detection of defective areas. To make this type of algorithm work, a separate database of damage profiles must be created to compare damage profiles throughout processing, which must be done for different conditions [22].

Defect evaluation and classification will be faster as a result, decreasing the future demand for computer processing power. Using this data to update the support life cycle model will provide accurate data and determine the remaining service life as well as the areas that are most prone to wear and tear [23].

The processing procedure presented needs to be tailored to machine learning software [24] so that the failure statistics of the catenary supports can be accumulated and the resulting models can be trained more accurately.

\section{Conclusions}

1. Centrifugal reinforced concrete supports are the most common type of support structure in the Russian railway catenary system, and their condition must be monitored continuously. 
2. While in service, reinforced concrete catenary supports are affected by series of factors contributing to defects and, consequently, decreased load-bearing capacity of the supports.

3. The effect of natural and climatic factors on the cracking of reinforced concrete catenary supports should be assessed.

4. The resulting inspection procedure will facilitate the processing automation of diagnostic measurements of catenary supports, reducing the time it takes to obtain accurate defect cards of supports, as well as cutting inspection costs and preventing any potential accidents caused by falling supports.

\section{References}

1. I.V. Ignatenko, S.A. Vlasenko, IOP Conf. Ser.: Earth Environ. Sci. 666, 032012 (2021)

2. V.N. Li, L.S. Demina, S.A. Vlasenko, E.Y. Tryapkin, IOP Conf. Ser.: Mater. Sci. Eng. 918, 012118 (2020)

3. K. Kobayashi, N. Banthia, J. Civ. Struct. Health Monit. 1, 25-35 (2011)

4. I.V. Ignatenko, S.A. Vlasenko, IOP Conf. Ser.: Mater. Sci. Eng. 918, 012154 (2020)

5. D. Schlicke, K. Hofer, N.V. Tue, Advanced Techniques for Testing of Cement-Based Materials (Springer, Cham, 2020)

6. M. Tsunemoto, M. Shimizu, Y. Kondo, T. Kudo, H. Ueda, T. Ijima, Q. Rep. RTRI 58, 270-276 (2017)

7. I. Ignatenko, E. Tryapkin, S. Vlasenko, A. Onischenko, V. Kovalev, Advances in Intelligent Systems and Computing 1115, 117-127 (2020)

8. D.V. Sannikov, A.S. Kolevatov, V.P. Vavilov, M.V. Kuimova, Appl. Sci. 8, 222 (2018)

9. P.K. Frankowskib, R. Sikora, T. Chadya, AIP Conf. Proc. 1706, 090008 (2016)

10. X. Zhao, P. Gong, G. Qiao, J. Lu, X. Lv, J. Ou, Sensors 11, 10798-10819 (2011)

11. Y. Bao, M. Valipour, W. Meng, K. Khayat, G. Chen, Smart Mater. Struct. 26, 085009 (2017)

12. H. Ueda, J. Adv. Conc. Tech. 18, 27-38 (2020)

13. V. Li, L. Demina, S. Vlasenko E3S Web Conf. 164, 03028 (2020)

14. C.A. Apostolopoulos, K.F. Koulouris, A.C. Apostolopoulos, Adv. in Civ. Eng. 2019 3438743 (2019)

15. B. Szymanik, P. Karol-Frankowski, T. Czady, C. Chelliah, Sensors 16, 234 (2016)

16. M. Rozli, C. Safiuddin, M. Harun et al., IOP Conf. Ser.: Mater. Sci. Eng. 615, 012123 (2019)

17. I.I. Sakharov, V.N. Paramonov, S.A. Kudryavtsev, Lecture Notes in Civil Engineering 49, 19-24 (2020)

18. C. Maierhofer, H. Reinhardt, G. Dobmann, Non-destructive testing methods (Woodhead Publishing, 2010)

19. W.I. Hamad, J. Phys.: Conf. Ser. 305, 012037 (2011)

20. E.Y. Romanenko, M.A. Trubitsin, Materials Science Forum, 931, 346-351(2018)

21. W. Li, S. Fan, S.C. Ho, J. Wu, G. Song, Structural Heal. Mon. 17, 461 - 471 (2018)

22. G. Li, Q. Liu, S. Zhao, W. Qiao, X. Ren, Meas. Sci. Technol. 31, 075403 (2020)

23. S. Laflamme, Meas. Sci. Technol 29(3), 1-12 (2018) 
24. A.N. Kobylitsky, I.V. Ignatenko, S.A. Vlasenko et al., IOP Conf. Ser.: Mater. Sci. Eng. 918, 012185 (2020) 